\title{
Der Politikstil von Jens Spahn - Von Konsensorientierung zu Konfliktbereitschaft
}

NILS C. BANDELOW, FLORIAN ECKERT, JOHANNA HORNUNG, ROBIN RÜSENBERG

Prof. Dr. Nils C. Bandelow ist Inhaber des Lehrstuhls für Vergleichende Regierungslehre und Politikfeldanalyse, TU Braunschweig

Dr. Florian Eckert ist Director Public Affairs bei fischerAppelt, Berlin

Johanna Hornung ist wissenschaftliche Mitarbeiterin am Lehrstuhl für Vergleichende Regierungslehre und Politikfeldanalyse, TU Braunschweig

Robin Rüsenberg ist Lehrbeauftragter am Lehrstuhl für Vergleichende Regierungslehre und Politikfeldanalyse, TU Braunschweig

Im Vergleich zu seinen Vorgängern zeichnet sich Jens Spahn durch eine außerordentliche Reformaktivität aus. Die Maßnahmen folgen aber keiner bisher erkennbaren inhaltlichen Vision. Sie fördern Bekanntheit und Beliebtheit des Ministers, werden aber von vielen sektoralen Akteuren kritisch gesehen. Unter dem Schlagwort „viel Taktik, wenig Strategie“ lassen sich vier Stilelemente subsumieren: Eine durchsetzungsfähige, aber nur teilweise gesundheitspolitisch ausgerichtete strategische Kernexekutive, die Nutzung individueller Expertise des Ministers in Verbindung mit ausgewählter Fachkompetenz, eine verstärkte Öffentlichkeitsorientierung mit besonderem Blick auf die Wirkung des Ministers und die ausgeprägte Konfliktfreudigkeit zur Überwindung von politischem Widerstand.

\section{Ministerielle Politikstile in der deutschen Gesundheitspolitik}

Seit der Umstrukturierung 1991 erlebt das heutige Bundesministerium für Gesundheit (BMG) mit Jens Spahn seit dem 14. März 2018 seine achte ministerielle Führung. Der auch über seine aktuelle Rolle hinaus ambitionierte Minister (Bröcker, 2018) sticht nicht nur aufgrund seiner vergleichsweisen frühen Karriere hervor. Bereits nach einem Jahr Amtszeit wird er als „Aufmischer“ mit eigenem Politikstil beschrieben (Beerheide, 2019). Nach der systematischen Abarbeitung des Koalitionsvertrags mit wenig öffentlicher Aufmerksamkeit unter seinem Vorgänger Hermann Gröhe hat Jens Spahn auch das Ministerium und gesundheitspolitische Themen wieder stärker ins Rampenlicht gebracht. Mehr noch als die konkreten Inhalte steht dabei die Person des Ministers in der öffentlichen Aufmerk- samkeit. Im Gegensatz zu seinen Vorgängern konnte Jens Spahn bisher persönlich von der Rolle des Gesundheitsministers profitieren. Dies zeigen nicht nur Beliebtheitsbefragungen in der Bevölkerung. Auch die Kanzlerin lobt Spahns aktive Rolle im Kabinett (Schwilden, 2019).

Die Vorgänger von Jens Spahn sind in mehrfacher Hinsicht von ihm zu unterscheiden. Das offensichtlichste Differenzierungsmerkmal ist die hohe Eigeninitiative. Durch die mit jeder Legislaturperiode zunehmende Bedeutung von Koalitionsverträgen sind die Minister immer mehr an vorab zwischen den Parteien verhandelte Kompromisse gebunden. Die zunehmende Länge und Detailtiefe der Verträge führt zu einer stärkeren Prägung und Prädiktion von Politik in einer Legislaturperiode und nicht immer sind die späteren „ausführenden" Ministerinnen und Minister an den Verhandlungen beteiligt und haben 
entsprechend die Chance, sich in „ihrem“ Politikfeld ein eigenes Profil zu machen (Bandelow, Eckert, \& Rüsenberg, 2017). Bereits Hermann Gröhe hatte ein hohes Arbeitspensum bei der Abarbeitung des 2013 ausgerechnet von Jens Spahn ausgehandelten Koalitionsvertrags (Knieps, 2016, p. 30). In der Vergangenheit hatten Ministerinnen und Minister mehr eigene Möglichkeiten der Gestaltung; innovative und komplexe Reformen wurden aber auch mangels ressortspezifischer Detailkenntnisse - häufig mithilfe einer in der Administration und Selbstverwaltung verankerten programmatischen Gruppe entwickelt und durchgesetzt (Hornung \& Bandelow, 2018).

Spahn schafft den Spagat zwischen beidem - Umsetzung des Koalitionsvertrags und dem Setzen eigener Akzente. Angesichts dieser im Vergleich zu vorherigen Amtsinhabern hohen Taktung an Politikproduktion stellt sich die Frage, wie sich der neue Politikstil von Jens Spahn theoretisch erklären und empirisch bestätigen lässt. Gibt es eine zugrundeliegende Strategie mit der er Vorschläge auf die Agenda bringt, selbst wenn diese keine unmittelbare Erfolgsaussicht haben? Der Beitrag beantwortet diese Fragen mit einer Analyse des Politikstils von Jens Spahn, die auf Medienberichten und anderen Veröffentlichungen sowie Experteninterviews basiert.

\section{Kriterien für optimierte Reformprozesse}

Die Identifizierung eines Politikstils und die Frage nach erfolgreichen Reformstrategien sind theoretisch miteinander verbunden. Stellt man sich den politischen Prozess als Kreislauf vor, der vom Setzen eines Themas auf die Agenda über die Formulierung und Verabschiedung bis hin zur Umsetzung und Evaluation von Politikergebnissen reicht, so ist die Art und Weise, wie diese einzelnen Schritte der Politikproduktion von Akteuren vollzogen werden, ausschlaggebend für deren Erfolg. Zentral sind dabei vier Fragen: Wie werden Themen gesetzt und politische Ideen entwickelt? Welche Akteure sind an der Entwicklung und Umsetzung konkreter Maßnahmen beteiligt? Wie werden diese kommuniziert? Wie werden Reformvorschläge durchgesetzt? Zur Optimierung von Reformprozessen hat die Bertelsmann Stiftung in Kooperation mit Politikwissenschaftlern ein Strategietool für politische Reformprozesse (SPR) entwickelt, das Erfolgskriterien für die einzelnen Fragen und unterschiedlichen Phasen im Politikprozess aufzeigt (Fischer, Kießling, \& Novy, 2008). In der Weiterentwicklung zu einem Strategie-Kompass werden diese in drei „K“s eingeteilt: Kompetenz, Kommunikation und Kraft zur Durchsetzung (Bertelsmann Stiftung, 2010). Damit

\section{Mit Blick auf das gesundheitspolitische Entscheidungszentrum der ersten Jahre unter Jens Spahn fällt die starke Rolle des Leitungsbereichs auf.}

im Bereich der Entscheidung und Umsetzung. In anderen Phasen - etwa dem Agenda-Setting oder der Politikformulierung ist die formalisierte Berücksichtigung dieser Akteure schwächer. Grundsätzlich stellt sich für die Exekutive immer die Frage, welche Gruppen in welchem Maß am politischen Prozesse beteiligt sein sollen.

Das Vorhandensein einer strategisch denkenden Kerngruppe, die zentrale Akteure und Entscheidungsträger vereint, ist jedoch noch nicht allein ausschlaggebend für den Erfolg von Reformen. Dieser ist auch abhängig davon, inwieweit die Kernexekutive bei der Entwicklung von Ideen auf externen wissenschaftlichen Sachverstand zurückgreift und wie sie

politische Ideen den Politikzyklus erfolgreich durchlaufen, benötigt es demnach eine strategische Kernexekutive, eine entscheidungsfähige aber inklusive Gruppe, die sich diesen Dreiklang zu eigen macht. Ein frühes Anwendungsfeld, das zur Entwicklung der Kategorien beigetragen hat, war das GKV-WSG (Bandelow \& Schade, 2008). Auch für die Analyse des Spahnschen Strategiestils eignet sich das Tool.

\section{Strategische Kernexekutive: I'État c'est Spahn - und sein Ministerium}

Der Politikstil in Deutschland zeichnete sich in der Vergangenheit durch eine konsensorientierte Einbindung von Stakeholder-Interessen aus, die jedoch durch die Veränderungen des Parteiensystems und der Notwendigkeit neuer Koalitionsmuster verändert wurde (Zohlnhöfer \& Tosun, 2019). Hintergrund eines institutionalisierten und konsensorientierten Austauschs zwischen den von Reformen betroffenen Stakeholdern ist, dass eine vorgelagerte Abstimmung untereinander die Chancen für die Reformdurchsetzung erhöht. Dabei ist zu beachten, dass diese strategische Kernexekutive ausreichend groß sein muss, dass alle Interessen berücksichtigt sind und gleichzeitig klein genug, um die Beibehaltung der Entscheidungsfähigkeit zu gewährleisten. Die strategische Kernexekutive ist institutionell verankert, bspw. durch regelmäßigem Austausch in entsprechenden formellen oder informellen Gremien oder Ideenzirkeln. Der vergleichsweise hohe Grad an Korporatismus in Deutschland bietet dabei eine institutionalisierte Einbindung ausgewählter Interessen, zumindest die Ideen gegenüber Stakeholdern und Bevölkerung kommuniziert. Dazu kommt auch die Herausforderung der Durchsetzung von Reformen. Zwar kann die frühzeitige Inklusion zentraler Akteure dem späteren Widerstand gegenüber neuen Bestimmungen entgegenwirken, aber auch die Umgangsformen sind bedeutsam. Mit Blick auf das gesundheitspolitische Entscheidungszentrum der ersten zwei Jahre unter Jens Spahn fällt zunächst die starke Rolle des zur Abteilung aufgewerteten Leitungsbereichs auf. Die zentralen Positionen der Leitungsabteilung wurden mit engen Vertrauten besetzt. Bis zur Neuordnung der Ministeriumsstruktur ab dem 1. Februar 2020 lag die Leitung bei Sonja Optendrenk, die durch ihre fachliche Erfahrung und das besondere Vertrauensverhältnis zum Minister eine zentrale Rolle einnimmt. Der mit der Neuordnung verbundene Wechsel dieser zentralen Mitarbeiterin an die Spitze der Abteilung 2 wird zu einer personellen Zusammenführung der Verantwortung für Gesundheitsversorgung und Krankenversicherung in der dafür vorgesehenen Fachabteilung führen. Die bisherige Leitungsabteilung verbindet dagegen fachliche Verantwortung mit anderen Aufgaben. Neben der Gesundheitspolitik war dies die strategische Profilierung des Ministers. Hier ist vor allem der stellvertretende $\mathrm{Ab}$ teilungsleiter Marc Degen verantwortlich, der 2018 phasenweise beurlaubt war, um die Kampagne des Ministers für den CDUParteivorsitz zu leiten.

Auch in der bisherigen Struktur wurden die jeweils zuständigen Abteilungsleiter und Unterabteilungsleiter fachlich eingebunden. Der Minister reagiert dabei we- 
niger als sein Vorgänger auf Vorschläge aus den Abteilungen, sondern konfrontiert die Fachebene selbst mit eigenen Anmerkungen etwa zu Stellungnahmen bei Anhörungen. In dem Austausch nimmt er auch pragmatisch Vorschläge aus dem Haus auf, versucht aber nicht zwingend Konsens mit seinen Mitarbeitern herzustellen. Auch die Verständigung mit den zentralen Interessengruppen des Gesundheitssystems - etwa Ärzten, Krankenkassen, Krankenhäusern, Apotheken, Pharmaindustrie und Patientenverbänden - erfolgt themenspezifisch unter aktiver Beteiligung Spahns und ist nicht zwingend auf Konsens ausgerichtet. Diese Abstimmung findet in wechselnden Koalitionen und stark themenspezifisch statt. Der Minister ist zudem ein Befürworter von transparenten und durchaus kritischen Debatten. Ihm wird Offenheit für gute Argumente nachgesagt - er scheut aber keine Konflikte, wenn es ihm nicht gelingt, alle relevanten Akteure für seine Positionen zu gewinnen.

Dass im alltäglichen politischen Geschäft die institutionalisierte Einbindung von Akteuren in exekutive Entscheidungen fehlt, heißt nicht, dass sie nicht punktuell durchaus vorhanden ist oder war. Ein Großteil von Spahns Politik besteht darin, die im Koalitionsvertrag verhandelten Punkte systematisch abzuarbeiten und jeweils noch eigene Akzente zu setzen. Die Koalitionsverhandlungen wurden von Unionsseite unter Führung der vorherigen Ministeriumsleitung (Hermann Gröhe und Lutz Stroppe) geführt und spiegeln den konsensorientierten Stil der vorigen Legislaturperiode. So wurde mit der Konzertierten Aktion Pflege im Koalitionsvertrag die Einrichtung einer Arbeitsgemeinschaft beschlossen, die in enger Zusammenarbeit zwischen dem BMG, Kassenverbänden, Berufsverbänden und Trägern pflegender Einrichtungen einen Maßnahmenkatalog für die Pflegepolitik ausarbeitete.

Insgesamt zeigt sich das Entscheidungszentrum im Ministerium als äußerst effizient und entscheidungsstark. Mit der Leitungsabteilung wurde zunächst ein Kern enger Vertrauter etabliert. Durch personelle und strukturelle Wechsel ist denkbar, dass die Zusammenarbeit zwischen diesem engen Kreis und der weiteren Expertise des Hauses noch weiter vertieft wird. Dies deutet darauf hin, dass es dem Minister vergleichsweise schnell gelingt, sich in dem Ministerium zu verwurzeln.

\section{Asymmetrische Experteneinbindung}

Die Entwicklung von innovativen politischen Programmen ist durch ihre fortschrittliche Orientierung auf neue wissenschaftliche Erkenntnisse angewiesen. Der Rolle von wissenschaftlicher Beratung ist in der internationalen Literatur viel Forschung gewidmet und wird vor allem in föderalen Mehrebenensystemen (Howlett \& Newman, 2010) als auch in korporatistischen Settings viel Gewicht beigemessen (Hustedt \& Veit, 2017). Eine systematische Experteneinbindung wird im Gesundheitswesen über verschiedene Beratungsgremien ermöglicht, auf die das Gesundheitsministerium zugreifen kann. Wissenschaftlicher Sachverstand bestimmt unter dem Stichwort der evidenzbasierten Medizin aktuell in vielen Ländern die Methodenbewertung (Kislov et al., 2019; Vecchione \& Parkhurst, 2015).

Das Verhältnis des Ministers zur Evidenzbasierung scheint allerdings nicht vollständig geklärt. So hat sich Jens Spahn mit den Vorstößen zur beschleunigten Methodenbewertung im G-BA aus Sicht von Beobachtern teilweise von der harten Evidenzbasierung gelöst (Wunderlich, 2019). Andere Evidenz, die auf Umstrukturierungen in der Steuerung des Gesundheitswesens zielen, werden dagegen in Gesetzesvorschläge eingebunden. So hat etwa das vom Sachverständigenrat zur Begutachtung der Entwicklung im Gesundheitswesen (SVR-G) angefertigte Gutachten zur bedarfsgerechten Steuerung der Gesundheitsversorgung sich auch mit der Notfallversorgung beschäftigt und entsprechende Lösungsvorschläge sind aktuell im Referentenentwurf befindlichen Gesetz zur Reform der Notfallversorgung aufgenommen. Nicht ganz zufällig wird sich der SVR-G nun mit einem Gutachten zur Digitalisierung von Gesundheit als Herzensthema von Jens Spahn beschäftigen. Der Sachverständigenrat wird zwar vom Minister ernannt und arbeitete zuletzt auf der Basis von Aufträgen des Ministeriums, er nutzt auch Räume und Ressourcen des BMG. Der SVR-G betont aber seine wissenschaftliche Unabhängigkeit und kann auf anerkanntes Renommee seiner Mitglieder zurückgreifen. Er berichtet auch explizit nicht nur an das Ministerium, sondern wendet sich auch an andere Akteure und die Öffentlichkeit. Dies gilt insbesondere für die Regionalkonferenzen und das abschließende Symposium zur Diskussion des Gutachtens von 2018.
Im deutschen Gesundheitswesen findet medizinischer, gesundheitsökonomischer und juristischer Sachverstand auch an anderen Stellen Berücksichtigung. Dies zeigt sich unter anderem an den weiterhin regelmäßig beauftragten wissenschaftlichen Gutachten. Teilweise krankt die Einbindung wissenschaftlicher Expertise aber an der geringen Zahl jeweils anerkannter einschlägiger Personen. Neben der „Fachlichkeit“ (die bisher in Deutschland auf Medizin, Rechtswissenschaft und Gesundheitsökonomie beschränkt wird) führen persönliche Vertrauensbeziehungen und die Verfügbarkeit von exklusiven Datensätzen zu einer starken Reduktion des eingebundenen Expertenkreises. Dies gilt etwa im Bereich der Pflegepolitik oder Honorarreform, in denen vor allem wenige ausgewiesene Spezialisten mit vielen Gutachten auftreten. Für den Kernbereich von Jens Spahns Amtszeit, Digitalisierung, hat er mit dem Health Innovation Hub (hih) seinen eigenen Think Tank eingerichtet. Dessen Leiter Jörg Debatin war auch schon Mitautor des Ministers zu diesem Thema (Spahn, Müschenich, \& Debatin, 2016). Auch hier zeigt sich, dass persönliches Vertrauen zentral für einen fruchtbaren Austausch zwischen Expertise und Entscheidung ist.

Während inhaltliche Lösungen zumindest systematisch fachlich begleitet werden, gibt es bisher im deutschen Gesundheitswesen noch keine Tradition der systematischen Nutzung wissenschaftlicher Evidenz für die nachhaltige und erfolgreiche Gestaltung von Reformprozessen unter Berücksichtigung der im Gesundheitswesen besonderen Herausforderung der Implementation. So gibt es inzwischen zwar eine Vielzahl von wissenschaftlich ausgearbeiteten Reformvorschlägen, die Umsetzung dieser Vorschläge in die breitere Praxis erweist sich oft als hohe Hürde. Dabei sind auch Innovationskraft und systemverändernde Reformen im Gesundheitswesen wesentlich von wissenschaftlichen Erkenntnissen abhängig (Bandelow \& Hornung, 2019; Whitney, Tazim, Bajnok, Edwards, \& Rowan, 2014). Nicht zuletzt deshalb pochen Forscher zunehmend auf eine stärkere evidenzbasierte Politikformulierung, die sich aus Evaluationsergebnissen und Erprobungsszenarien speist (Boswell, 2018; Cairney \& Oliver, 2017). Gerade das Gesundheitswesen als staatsnaher und über Politik gesteuerter Sektor bedarf neben medizinischer, gesundheitsökonomischer, juristischer und 
pflegewissenschaftlicher Expertise auch der Nutzung der Sozialwissenschaften, die bisher nur über einzelne Akteure in das System einfließt. Politik- und Verwaltungswissenschaften treten oft nur in Erscheinung, wenn parteinahe Professoren politische Perspektiven mit teilweise geringem Bezug zu konkreter Forschung in den Medien legitimieren. Daneben bietet aber vor allem die wissenschaftliche politische Prozessforschung vielfältige methodische und theoretische Perspektiven sowie empirische Ergebnisse aus Deutschland und im internationalen Vergleich, um nicht nur die gesundheitspolitischen Inhalte, sondern auch die Steuerungsprozesse evidenzbasiert zu gestalten. Bezogen auf die berücksichtigten Disziplinen ist die Experteneinbindung daher weiterhin asymmetrisch.

Die bisher unsystematische Berücksichtigung von wissenschaftlichen Erkenntnissen - insbesondere der Verwaltungs- und Politikwissenschaft - in seinen Reformen liegt zum Teil darin begründet, dass sich der Gesundheitsminister im Gegensatz zu vielen seiner Vorgänger selbst bereits ein fundiertes Wissen über das Gesundheitssystem angeeignet hat (Szent-Ivanyi, 2018). Ein Alleinstellungsmerkmal des langjährigen gesundheitspolitischen Sprechers der CDU/CSU-Fraktion ist damit sein biographischer Werdegang als Politologe und die mit den vorherigen Positionen verbundene Fachexpertise. Fachspezifische Expertise stellt zwar kein notwendiges Kriterium für das Bekleiden eines entsprechenden Ministerpostens dar, und sozial- bzw. gesundheitspolitischer Sachverstand im Ministeramt erklärt nicht unbedingt die Art und den Umfang von Gesetzen - nicht zuletzt aufgrund einer gewissen Gestaltungsmöglichkeit durch die Ministerialbürokratie. Das Vorhandensein derselben ist aber durchaus hilfreich und nahezu unverzichtbar für die Entwicklung inhaltlicher Programme und tiefgreifender Reformvorschläge sowie deren Durchsetzung.

\section{Kommunikation: Die Marke Spahn}

Vor allem für die Gesundheitspolitik hat sich ein entsprechender Frame, Narrativ bzw. eine Storyline für die Durchsetzung von Reformen als enorm wichtig erwiesen (Crespy \& Szabó, 2018). Die politische Prozessforschung empfiehlt folglich die Nutzung systematisch entwickelter Narrative zur Legitimation, die weit über die verbreitete Reformkommunikation hinaus- geht: Erfolgreiche Narrative basieren unter anderem auf einer klaren Benennung (oder Erzeugung) von Problemwahrnehmungen, die im Vorfeld von Reforminitiativen von einem Großteil der Bevölkerung geteilt werden müssen. Reformkommunikation, die direkt an Instrumenten ansetzt (etwa "Wettbewerb" oder "Digitalisierung") ist gerade im Hinblick auf die Umsetzung umfassender Veränderungen nicht erfolgversprechend. Die Gesundheitspolitik gilt nach wie vor als heikles Thema, mit dem Wahlen verloren, aber nicht gewonnen werden können (Knieps, 2016, p. 27). Daher muss die Kommunikation gesellschaftlichen Problemdruck aufnehmen, Emotionen erzeugen und Lösungen so präsentieren, dass sie über die klassischen Konfliktlinien hinaus akzeptiert werden. Die Kommunikation von politischen Vorhaben mit einer klaren Benennung von identifizierten Problemen und der Art, wie die Reformvorschläge diese lösen können, trägt auch zur Legitimation der Reformen gegenüber Interessengruppen bei. Bei diesen ist es besonders wichtig, dass diese sich mit dem Narrativ identifizieren können; dass sie in den kommunizierten Frames also ihre bestehenden Interessen und Werte wiederfinden. Das bedeutet nicht, dass die Interessen tatsächlich durch die Reform bedient werden - angesichts der Vielfalt an gesundheitspolitischen Akteuren ist es wohl auch kaum möglich, dass eine Reform allen Interessen gerecht wird. Um die Akzeptanz zu erhöhen muss aber die Kommunikation der Reform so gestaltet sein, dass sie gesichtswahrend für die Akteure ist und alle einen für sich positiven Aspekt der Reform sehen können.

Jens Spahn kommuniziert viel. Was er kommuniziert ist aber nicht immer das, was Reformerfolg ausmacht. In der Öffentlichkeit ist er als aktiver Minister aufgefallen, dessen Beliebtheitswerte verglichen mit anderen Ministerinnen und Ministern gestiegen sind (Mestermann, 2019). Wenn man sich die Reforminhalte genauer ansieht, fehlt aber ein $\mathrm{Zu}$ sammenhang zwischen den Reformen, der auf ein ideengestütztes, innovatives Programm hindeutet. Stattdessen findet sich eine Vielzahl an Gesetzen, die besonders öffentlichkeitswirksam sind, deshalb kurzfristig gut vermarktet werden können und für Spahn Bekanntheit und Beliebtheit bringen. Vielfach kann er sich dabei auf grundlegende Formulierungen des Koalitionsvertrags berufen, dessen Vorgaben er aber oft mit eigenen Initiativen für sich nutzt. Dazu gehören das Organspendegesetz (mit dem letztlich nicht erfolgreichen Streben nach einer Widerspruchslösung), der LipödemVorstoß rund um die G-BA-Methodenbewertung, das Masernschutzgesetz und das Terminservice- und Versorgungsgesetz, durch das die Wartezeiten auf Facharzttermine vor allem für Kassenpatienten verkürzt werden sollen. Die Reformaktivität scheint deshalb teilweise auf die Platzierung der „Marke Spahn“ gerichtet zu sein und weniger auf das visionäre, langfristige Lösen von Problemen im Gesundheitswesen.

Das fehlende zusammenhängende Narrativ, das seine Reformen kommunikativ verbindet und damit Probleme mit von ihm gebotenen Lösungen verknüpft, kann sich dabei allerdings auch zum Bumerang für 
bereits im Vorfeld mit möglichst vielen betroffenen Akteuren über Reformen verständigt hat. Aus einer prozessualen und weniger institutionellen Sicht ist es aber ebenso relevant, wie die formal entscheidungsfähige Kerngruppe die Reformen gegenüber anderen Akteuren stilistisch durchsetzt, wie also auch hier der Kooperationsstil ausgestaltet ist.

Die Beteiligung der Stakeholder, die bei den vorangegangenen Kriterien für erfolgreiche Reformprozesse bereits als unzureichend festgestellt wurde, setzt sich auch mit Blick auf die prozessuale Durchsetzung von Reformen fort. Jens Spahn zeichnet sich durch eine beeindruckende Konfliktfähigkeit aus, die von einigen Beobachtern sogar als Konfliktfreudigkeit als Persönlichkeitsmerkmal beschrieben wird. Demnach sucht er auch aktiv nach Konflikten, vor allem mit der Selbstverwaltung, die er wie oben erwähnt gerne als offene und kritische Debatten bezeichnet, um Aufmerksamkeit zu erzeugen und zu provozieren. Das Verhältnis zu den Stakeholdern unterscheidet sich damit diametral von dem konsensorientierten Politikstil Hermann Gröhes (Knieps, 2018, p. 6f), der um maximale Konsensorientierung bemüht war.

Der Fokus liegt dabei oft, aber nicht nur auf der Selbstverwaltung, die der Untätigkeit und Verwässerung des politischen Willens verdächtigt wird. Zwar sind staatliche Eingriffe in den Kompetenzbereich der Selbstverwaltung viel kritisiert (Egger, 2019), diese sind aus Sicht des Ministers aber kein Selbstzweck, sondern zielen darauf, dass Prozesse funktionieren.

Sowohl zur AG Gesundheit der CDU/ CSU-Bundestagsfraktion als auch zur gesundheitspolitischen Spitze der SPD-Bundestagfraktion wird ein enger Austausch über die Agenda des BMG gesucht - über die konkrete Ausgestaltung der politischen Maßnahmen in den Gesetzesentwürfen besteht dann aber bei den Gesundheitspolitikern der Regierungsfraktionen oftmals Überraschung (ironischerweise eine Vorgehensweise, über die sich Spahn in seiner eigenen Zeit als gesundheitspolitischer Sprecher immer geärgert hatte). Vor allem die Quantität der Reformvorschläge sowie die enge zeitliche Taktung innerhalb der Gesetzgebungsprozesse mit zahllosen (auch sachfremden) Änderungsanträgen stellt die personellen Kapazitäten in den Fraktionen auf eine harte Probe - und stärken im Ergebnis die Stellung des Ministeriums mit seiner fachlichen Expertise.
Jens Spahn fährt Konflikte aber nicht (nur) aus Lust an der Konfrontation. Wenn es Probleme gibt, wie etwa bei der Digitalisierung von Gesundheitsleistungen, schreckt er nicht davor zurück, die Mehrheitsverhältnisse in der gematik zu ändern und sich selbst den Problemen zu widmen. Radikale Forderungen wie dem bisher gescheiterten Vorschlag der bundesweiten Öffnung von AOKen interpretieren viele Beobachter als gezielte taktische $\mathrm{Ab}$ lenkung zur Durchsetzung anderer Ziele.

\section{Fazit: Der Politikstil von Jens Spahn}

Der Politikstil von Jens Spahn unterscheidet sich schon auf den ersten Blick von dem seiner Amtsvorgänger. Auf den zweiten Blick hat die in diesem Beitrag auf Basis des Strategietools für politische Reformprozesse durchgeführte Analyse tiefere Einblicke darin gegeben, welche Kernelemente den ministeriellen Politikstil ausmachen. Anhand der vier Kriterien für erfolgreiche Reformdurchsetzung lassen sich abschließend vier Stilkomponenten des Ministers erkennen.

Die erste bezieht sich auf Verbindung gesundheitspolitischer Ziele mit den eigenen Karriereorientierungen schon bei der Zusammensetzung der bisher zentralen Leitungsabteilung. Akteure innerhalb und außerhalb seines Hauses werden in fachlich wechselnden Konstellationen gehört, ohne dabei zwingend konsensuale Lösungen anzustreben.

\section{Der Politikstil von Jens Spahn lässt sich als „viel Taktik, wenig Strategie" zusammenfassen.}

der Bevölkerung aus. Es ist kein Geheimnis, dass er auf lange Sicht das Kanzleramt anstrebt. Darunter leidet die Kommunikation seiner gesundheitspolitischen Strategie. Zwar wird der Kommunikation einzelner Reformen in den Medien und auch in der Fachwelt ein hohes Maß an Aufmerksamkeit gewidmet, es fehlt aber an einer Verbindung und zusammenhängenden Kommunikation von Problemen und dafür identifizierten Lösungen, die auch nachhaltig das Gesundheitswesen optimieren und umstrukturieren können. Die Abstimmung mit den Stakeholdern und der Umgang mit ihnen bei der Durchsetzung neuer Gesetze ist von hohem Tempo geprägt sowie z. T. provokant und greift mitunter bestehende Strukturen der Selbstverwaltung an. Für die Stakeholder in diesen Bereichen ist dieser Umgang teilweise befremdlich; ihnen fehlt bei Spahn der echte Wille, das Gesundheitswesen unter Einbeziehung von der im Sektor vorhandenen Expertise tiefgreifende strategische Politik zu betreiben.

Am Ende lässt sich der Politikstil insgesamt als „,viel Taktik, wenig Strategie“ zusammenfassen. Die vielen Gesetzesentwürfe sind insgesamt überwiegend erfolgreich. Trotz einzelner Niederlagen gelingt es Jens Spahn im Gegensatz zu seinen Amtsvorgängern, das Gesundheitsressort für seine persönliche politische Profilierung zu nutzen. Die wesentlichen Probleme des Gesundheitswesens (vor allem Fachkräftemangel, nachhaltige Finanzierung, ländliche Gesundheitsversorgung, Überwindung von Systemgrenzen innerhalb des Gesundheitswesens, Umgang mit Fehlanreizen für Akteure des Systems) wurden dabei nur zum Teil gelöst. Die bisherigen

Damit verbunden ist das zweite Stilelement: Vertrauen in die eigene Fachexpertise. Die im Gesundheitswesen zentralen Experten aus Medizin, Rechtswissenschaft und Ökonomie werden zwar weiterhin mit zahlreichen Gutachten und Studien beauftragt. Die faktische Nutzung der Expertise für Entscheidungen erfolgt aber unsystematisch in Abhängigkeit von der jeweils eigenen Bewertung des Ministers. Das dritte und wohl auffälligste Stilelement von Spahns Politik ist die Eigenvermarktung. Die hohe Reformaktivität und das Aufgreifen von emotionalisierenden und öffentlichkeitswirksamen Themen wie Impfen und Organspende zahlen sich bisher für die Beliebtheit des Ministers in
Lösungen zeichnen sich zudem dadurch aus, dass sie auf der aktuell sehr guten Finanzlage der GKV fußen. Nachhaltige Reformen mit Einschnitten zu Lasten starker Interessengruppen bedürfen eines umfassenden Narrativs, das etwa vergleichbar mit der Klimapolitik Reformbereitschaft durch die zusammenhängende Bezeichnung von Problemen, Opfern, Tätern und Lösungen generiert. Hier bleibt abzuwarten, ob die zusätzlichen Freiheiten nach der Umsetzung eines großen Teils des Koalitionsvertrags, der verstärkten Verankerung im Ministerium und dem Rückhalt durch Umfrageergebnisse zu einer strategischen Erweiterung des Politikstils führen. 


\section{Literatur}

Bandelow, N. C., Eckert, F., \&

Rüsenberg, R. (2017). Wie funktionieren

Koalitionsverhandlungen in der

Gesundheitspolitik? MIS - Management

Information System Gesundheitspolitik.

https://observer-gesundheit.de/wie-

funktionieren-koalitionsverhandlungen-dergesundheitspolitik/ (zuletzt eingesehen am 14. Februar 2020)

Bandelow, N. C., \& Hornung, J. (2019). Mehr Staat, weniger Selbstverwaltung, weniger Wettbewerb? Mut zur Evidenz - auch bei Governance-Fragen! Observer Gesundheit, retrieved from: https://observer-gesundheit. de/mehr-staat-weniger-selbstverwaltungweniger-wettbewerb-mut-zur-evidenz-auchbei-governance-fragen/.

Bandelow, N. C., Hornung, J., \& Iskandar, L. Y. (2020). Warum Jens Spahns Digitalisierungsstrategie kränkelt. Observer Gesundheit https:// observer-gesundheit.de/warum-jens-spahnsdigitalisierungsstrategie-kraenkelt/ (zuletzt eingesehen am 21. Januar 2020).

Bandelow, N. C., \& Schade, M. (2008). Die Gesundheitsreform der Großen Koalition: Strategische Erfolge im Schatten des Scheiterns. In T. Fischer (Ed.), Politische Reformprozesse in der Analyse (pp. 85-144). Gütersloh: Verl. Bertelsmann-Stiftung.

Beerheide, R. (2019). Ein Jahr Jens Spahn: Aufmischer der Gesundheitspolitik. Ärzteblatt, $116(10)$, A-457 / B-375 / C-371.

Bertelsmann Stiftung. (2010). Die drei „K“ einer erfolgreichen Strategie. In B. Stiftung (Ed.), Mehr Strategie wagen. Strategie-Kompass für politische Reformprozesse (pp. 12-16). Gütersloh: Verlag Bertelsmann Stiftung.

Boswell, J. (2018). What Makes Evidence-Based Policy Making Such A Useful Myth? The Case of NICE Guidance on Bariatric Surgery in the United Kingdom. Governance, 31(2), 199-214. doi:10.1111/gove.12285

Bröcker, M. (2018). Jens Spahn: Die Biografie. Freiburg im Breisgau: Verlag Herder $\mathrm{GmbH}$.

Cairney, P., \& Oliver, K. (2017). Evidence-based Policymaking is not like Evidence-based Medicine, So How Far Should You Go to Bridge the
Divide Between Evidence and Policy? Health Research Policy and Systems, 15(35), 1-11.

Crespy, A., \& Szabó, I. (2018). Healthcare Reforms and Fiscal Discipline in Europe: Responsibility or Responsiveness? European Policy Analysis, 4(2). doi:10.1002/epa2.1041

Egger, B. (2019). Staatliche Eingriffe in den Leistungskatalog der GKV - Selbstverwaltung auf Abruf? G\&S Gesundheits- und Sozialpolitik 73(4-5), 8-13. doi:10.5771/1611-5821-2019-4-5-8

Fischer, T., Kießling, A., \& Novy, L. (2008). Einführung in die Konzeption des Strategietools für politische Reformprozesse (SPR). In T. Fischer, A. Kießling, \& L. Novy (Eds.), Politische Reformprozesse in der Analyse. Untersuchungssystematik und Fallbeispiele (pp. 9-84). Gütersloh: Verlag Bertelsmann Stiftung.

Hornung, J., \& Bandelow, N. C. (2018).

The Programmatic Elite in German Health Policy: Collective Action and Sectoral History. Public Policy and Administration. doi:10.1177/0952076718798887

Howlett, M., \& Newman, J. (2010). Policy Analysis and Policy Work in Federal Systems: Policy Advice and Its Contribution to Evidence-Based Policy-Making in Multi-Level Governance Systems. Policy and Society, 29(2), 123-136. doi:10.1016/j.polsoc.2010.03.004

Hustedt, T., \& Veit, S. (2017). Policy Advisory Systems: Change Dynamics and Sources of Variation. Policy Sciences, 50(1), 41-46. doi:10.1007/s11077-016-9272-y

Kislov, R., Wilson, P., Cummings, G., Ehrenberg, A., Gifford, W., Kelly, J., . . . Harvey, G. (2019). From Research Evidence to "Evidence by Proxy"? Organizational Enactment of Evidence-Based Health Care in Four High-Income Countries. Public Administration Review, 79(5), 684-698. doi:10.1111/puar.13056

Knieps, F. (2016). Gesundheitspolitik zwischen Wettbewerb, Selbstverwaltung und staatlicher Steuerung - Versuch einer Bilanz der Gesundheitsreformen seit 1989. In E. Wille (Ed.), Entwicklung und Wandel in der Gesundheitspolitik. 20. Bad Orber Gespräche über kontroverse Themen im Gesundheitswesen (pp. 25-36). Frankfurt am Main: Peter Lang
$\mathrm{GmbH}$ - Internationaler Verlag der Wissenschaften.

Knieps, F. (2018). Kontinuität vor Wandel in der Gesundheitspolitik - Der Vertrag für eine erneute Große Koalition. Gesundheits- und Sozialpolitik, 2018(2), 6-9.

Mestermann, M. (2019). Klöckner und Altmaier sind Absteiger des Jahres. SPIEGEL Politik: https:// www.spiegel.de/politik/deutschland/peteraltmaier-und-julia-kloeckner-sind-absteiger-desjahres-regierungsmonitor-a-1301451.html (zuletzt eingesehen am 14. Januar 2020).

Schwilden, F. (2019). Der Wegschaffer. https://www.welt.de/politik/deutschland/ plus197540681/Jens-Spahn-Der-Gesundheitsminister-der-wegschafft.html (zuletzt eingesehen am 7. Januar 2020).

Spahn, J., Müschenich, M., \& Debatin, J. F. (2016). App vom Arzt. Bessere Gesundheit durch digitale Medizin. Freiburg im Breisgau: Verlag Herder $\mathrm{GmbH}$.

Szent-Ivanyi, T. (2018). Jens Spahn hat viel vor. Frankfurter Rundschau, https://www.fr.de/ politik/jens-spahn-viel-10994394.html (zuletzt eingesehen am 7. Januar 2020).

Vecchione, E., \& Parkhurst, J. (2015). The Use of Evidence Within Policy Evaluation in Health in Ghana: Implications for Accountability and Democratic Governance. European Policy Analysis, 1(2), 111-131. doi:10.18278/epa.1.2.6

Whitney, B., Tazim, V., Bajnok, I., Edwards, N. \& Rowan, M. (2014). Understanding Whole Systems Change in Health Care: Insights Into System Level Diffusion From Nursing Service Delivery Innovations - A Multiple Case Study. Evidence \& Policy, 10(3), 313-336.

Wunderlich, S. (2019). Evidenzbasierte Medizin als Spielball der Politik: Die zweite Runde. Observer Gesundheit https://observer-gesundheit. de/evidenzbasierte-medizin-als-spielball-derpolitik-die-zweite-runde/ (zuletzt eingesehen am 11. Januar 2020).

ZohInhöfer, R., \& Tosun, J. (2019). Policy Styles in Germany. Still Searching for the Rationalist Consensus? In M. Howlett \& J. Tosun (Eds.), Policy Styles and Policy-Making. Exploring the Linkages. New York: Routledge. 